\title{
COMO TREINAR SUA ABELHA: MÉTODOS APLICADOS À BIOLOGIA COGNITIVA DA POLINIZAÇÃO
}

\author{
João Marcelo Robazzi Bignelli Valente Aguiar ${ }^{1,2 *}$, Francismeire Jane Telles ${ }^{3}$, Pedro Joaquim \\ Bergamo $^{1}$, Vinicius Lourenço Garcia de Brito ${ }^{4} \&$ Marlies Sazima ${ }^{5}$
}

${ }^{1}$ Universidade Estadual de Campinas, Instituto de Biologia, Programa de Pós-Graduação em Ecologia, Av. Bertrand Russel, s/n, CP 6109, Cidade Universitária Zeferino Vaz, CEP 13083-865, Campinas, SP, Brasil.

${ }^{2}$ Université Paul Sabatier - Toulouse III, Centre de Recherches sur la Cognition Animale, Bât 4R3, 118 route de Narbonne, Toulouse, França.

${ }^{3}$ Universidade Federal de Uberlândia, Instituto de Ciências Biológicas, Programa de Pós-Graduação em Ecologia e Conservação de Recursos Naturais, Campus Umuarama, Bloco 1G, sala 43, CEP 38400-902, Uberlândia, MG, Brasil.

${ }^{4}$ Universidade Federal de Uberlândia, Instituto de Ciências Biológicas, Campus Umuarama, Bloco 1G, sala 43, CEP 38400-902, Uberlândia, MG, Brasil.

${ }^{5}$ Universidade Estadual de Campinas, Instituto de Biologia, Av. Bertrand Russel, s/n, CP 6109, Cidade Universitária Zeferino Vaz, CEP 13083-865, Campinas, SP, Brasil.

E-mails: jmrobazzi@gmail.com (*autor correspondente); meirecuesta@gmail.com; pjbergamo@gmail.com; viniciusduartina@gmail.com; marlies.sazima@gmail.com

Resumo: As abelhas são os principais vetores bióticos da polinização, fenômeno extremamente importante para a manutenção dos sistemas naturais e também para a produção de alimentos. Durante décadas, estudos mostraram como as abelhas são notáveis quanto às suas capacidades cognitivas através de protocolos desenvolvidos para se entender o comportamento, aprendizado e memória desses insetos. O objetivo desta revisão é apresentar as bases dos experimentos de cognição em abelhas, descrevendo protocolos clássicos, novas abordagens e estudos empíricos mostrando como o treinamento de abelhas pode ser aplicado à diferentes questões na biologia da polinização.

Palavras-chave: aprendizado; cognição; comportamento; memória; percepção.

HOW TO TRAIN YOUR BEE: METHODS APPLIED TO THE COGNITIVE BIOLOGY OF POLLINATION. Bees are the major biotic pollination agents, an extremely important phenomenon to the maintenance of the natural systems and food production. For decades, studies have shown the remarkable cognition system of bees, and several protocols were developed to understand behavior, learning and memory of these insects. The aim of this review is to present the basis of cognitive experiments using bees as a model, describing classical protocols, new approaches and empiric studies showing how bee training can be applied to different questions on pollination biology.

Keywords: behavior; cognition; learning; memory; perception. 


\section{INTRODUÇÃO}

A polinização é um importante processo ecológico, consistindo na transferência de grãos de pólen das anteras para o estigma das flores, dando início a fertilização dos óvulos e subsequente produção de frutos e sementes (Willmer 2011, Rech et al. 2014a). A transferência do pólen pode ocorrer por meio de vetores abióticos, como o vento e a chuva (Rech et al. 2014b) ou, mais comumente, por vetores bióticos: animais que ao visitarem as flores em busca de recursos florais (e.g., néctar) coletam, direta ou indiretamente, os grãos de pólen, carregando-os até as próximas flores (Rech et al. 2014a). Dentre os vetores bióticos encontram-se diversos grupos de animais, como beija-flores, borboletas, vespas, moscas e abelhas (Rech et al. 2014a). Estima-se que, globalmente, 87\% das Angiospermas dependem de animais para produção de sementes e nos trópicos esse número pode ultrapassar $90 \%$ (Ollerton et al. 2011, Rech et al. 2016).

Dentre os diferentes grupos de polinizadores, as abelhas merecem destaque, pois a grande maioria se alimenta exclusivamente dos recursos florais. $\mathrm{O}$ uso de diferentes capacidades sensoriais e cognitivas pelas abelhas têm importante papel no processo de forrageio nas flores (Giurfa 2007, Melo et al. 2018). Esses insetos são capazes de identificar as fontes de recurso utilizando sinais emitidos pelas flores, os chamados atrativos florais, como cores e fragrâncias, e aprendem a associar tais sinais com a presença ou ausência de recurso (i.e., néctar) (Giurfa 2007, Brito et al.2015). Quando o recurso compensa o esforço da visita (e.g., distância percorrida entre recurso e ninho, tempo de manipulação da flor, etc), as abelhas estabelecem uma rota de forrageamento para explorá-lo, permanecendo assim até que a necessidade da prole (i.e., pólen e néctar) e/ou do indivíduo (i.e., néctar) seja saciada ou mesmo até o fim da florada (Chittka et al. 1999). Dada essa dependência, as abelhas são interessantes modelos para entender o papel da sinalização floral e cognição animal no processo de polinização.

O entendimento da relação entre a capacidade sensorial e cognitiva das abelhas com os sinais e recursos florais começou há mais de um século. Um dos primeiros registros foi o experimento realizado por Charles Henry Turner (1910), mostrando pela primeira vez que indivíduos de Apis mellifera (Hymenoptera, Apidae) aprendiam a associar papel colorido com a presença de mel. No entanto, foi o experimento de Karl von Frisch (1914) que realmente demonstrou que abelhas eram capazes de perceber o mundo em cores. Para demonstrar a capacidade visual e cognitiva em abelhas, Frisch (1914) treinou indivíduos de A. mellifera para coletar soluções açucaradas associadas a um pedaço de cartolina azul. Através deste simples experimento associativo foi demonstrado que abelhas eram capazes de (i) aprender a associar o estímulo com o recurso oferecido e (ii) identificar o estímulo correto quando na presença de outros estímulos de mesmo tamanho, porém em vários tons de cinza (i.e., acromáticos), demonstrando que a seleção era baseada em sinais visuais coloridos, pois selecionaramoúnico estímulocromáticodisponível, independentemente da intensidade e posição espacial (Frisch 1914, Chittka et al. 2014). Esse experimento permaneceu "esquecido" até meados do século $\mathrm{XX}$, quando pesquisadores europeus retomaram o programa científico de entender os processos cognitivos em abelhas (Dyer et al. 2015). Ainda assim, muitos dos testes empregados para explorar aspectos cognitivos da relação entre flores e visitantes florais foram modificações do projeto inicial de von Frisch (Chittka et al. 2014). Atualmente, após décadas de experimentos e do estabelecimento das abelhas como modelo em estudo de aprendizado e memória animal, sabe-se que estes insetos possuem um diverso repertório cognitivo, sendo capazes de realizar tarefas que por muito tempo eram conhecidas apenas como realizáveis por vertebrados, incluindo humanos (Giurfa 2007).

As abelhas, especialmente as sociais, podem ser facilmente criadas e treinadas em cativeiro (Giurfa 2007). Ainda, é possível manipular tanto os estímulos como os recursos, pois ambos se encontram no mesmo alvo: as flores. De fato, o treinamento de abelhas é uma atividade simples, podendo ser realizado por crianças em atividades escolares (Blackawton et al. 2011). A maioria dos estudos foi conduzida com abelhas sociais dos gêneros Apis e Bombus (Weiss 2001, Chittka \& Thomson 2004), porém estudos recentes têm sido conduzidos com outras abelhas, como as Meliponini, as quais também podem ser facilmente treinadas em experimentos de comportamento (Henske et al. 2015, Koethe et al. 2016, Balamurali et al.2018). 
Nessa revisão, mostramos os conceitos básicos que devem ser considerados em estudos sensoriais e cognitivos com abelhas. Além disso, descrevemos o raciocínio e estrutura de alguns dos principais experimentos no contexto da biologia da polinização, revelando como essa abordagem é importante para entender o processo de polinização pela perspectiva dos polinizadores, e suas consequências para as plantas. Também apresentamos como material suplementar, um glossário de termos técnicos para uma melhor compreensão do texto (Material Suplementar 1).

\section{MATERIAL E MÉTODOS}

Essa revisão é baseada no conhecimento dos pesquisadores e na literatura com os temas cognição e condicionamento de abelhas. Selecionamos artigos teóricos e experimentais, livros texto, além de trabalhos sobre biologia da polinização utilizando as metodologias de ecologia cognitiva e treinamento de abelhas. Realizamos a pesquisa de forma não sistemática entre junho de 2018 e junho de 2019 nas bases de dados Web of Science e Google Scholar.

\section{CORES E FRAGRÂNCIAS: OS PRINCIPAIS SINAIS FLORAIS}

O primeiro passo para quem deseja estudar a biologia cognitiva da polinização é entender que os sinais florais não evoluíram em resposta ao sistema sensorial e cognitivo dos humanos. Estes sinais são resultado de seleção natural através das interações entre as flores e seus visitantes. Sinais florais relevantes para o sistema sensorial e cognitivo dos polinizadores podem estar sob seleção fenotípica mediada pelas abelhas (Sletvold et al.2016), evidenciando a natureza evolutiva desta relação. Além disso, as abelhas frequentemente estão expostas a sinais complexos e variáveis dependendo da comunidade de flores com a qual interagem (Chittka 1997). A regra geral é que a relevância de cada sinal floral estará estruturada dentro de um contexto, no qual o interagente e o ambiente podem representar forças seletivas (Telles et al. 2017, Bergamo et al. 2018). Como os polinizadores não percebem os sinais florais da mesma forma que os humanos, é necessário considerar esse aspecto tanto em observações de campo, quanto em experimentos cognitivos (Brito et al. 2014).

Muitos atrativos florais atuam na comunicação entre flores e polinizadores, como forma, cor e fragrância (Wester \& Lunau 2017, Melo et al. 2018). As cores representam um dos sinais mais conspícuos para a maioria dos visitantes florais, pois são percebidas de forma rápida e à longa distância, permitindo que os animais obtenham informações sobre o recurso mesmo antes da visita (Wester \& Lunau 2017). Para perceber a informação visual, as abelhas dispõem de um sistema composto por três ocelos, também chamados de olhos simples, responsáveis pela percepção de intensidade e direção da luz, além de olhos compostos, formados por centenas de unidades conhecidas como omatídeos, que permitem a visão em cores através dos fotorreceptores (Stavenga 1992, Land \& Nilsson 2002). A maioria das abelhas estudadas possui três tipos de fotorreceptores, com picos de excitação nas faixas do ultravioleta, azul e verde (Briscoe \& Chittka 2001).

A informação sobre morfologia, fisiologia, comportamento esistema neuronal para as espécies modelo de abelhas (A. mellifera e Bombus spp.), possibilitou o desenvolvimento de modelos de visão para as abelhas. Estes modelos nos permitem inferir a maneira como as abelhas enxergam as cores/padrões das flores (Gawryszewski 2018). Esses modelos são essenciais para, entre outras coisas, determinar se o estímulo utilizado em experimentos de aprendizado e memória é compatível com a cor da flor na natureza. De modo geral, estes modelos necessitam de quatro inputs básicos: a reflectância espectral do objeto que se deseja modelar a cor, as sensibilidades espectrais da espécie da qual se deseja modelar a visão (i.e., de cada tipo de fotorreceptor), a reflectância do plano fundo no qual o objeto está contrastado, e a iluminação do ambiente (Gawryszewski 2018).

Apesar dos modelos darem uma noção da percepção das cores pelas abelhas, é importante notar que o condicionamento experimental também pode melhorar a discriminação de cores pelas abelhas (Giurfa 2004). Além disso, como cada tipo de modelo visual diverge em suas premissas, o uso de um ou outro modelo deve ser cuidadosamente aplicado e justificado pelo pesquisador (Kemp et al. 2015, Telles \& RodríguezGironés 2015, Gawryszewski 2018). Portanto, estes 
modelos servem como uma primeira abordagem das capacidades visuais dos polinizadores. Seu uso também é interessante quando não é possível fazer experimentos comportamentais, por exemplo, quando se trabalha com um número elevado de espécies vegetais (Bergamo et al. 2018, Aguiar \& Pansarin 2019).

Diferentemente dos sinais visuais que são percebidos de maneira mais direta, a percepção da fragrância depende da dispersão pelo vento, podendo sofrer diluições e misturas com outras fragrâncias no ambiente até chegar ao polinizador (Wester \& Lunau 2017). As fragrâncias são combinações de diversos compostos voláteis que geralmente formam uma mistura única em cada espécie, a qual também pode variar entre indivíduos de uma população (Salzmann et al. 2007, Raguso 2008, Baldelomar et al. 2018). Por haver essa grande variação, a fragrância pode ser utilizada pelo polinizador para identificar flores específicas (Raguso 2008). As abelhas percebem as fragrâncias por meio de sensores químicos nas antenas, conectados ao lobo antenal, principal região codificadora de olfato no cérebro desses insetos (Giurfa 2007). Experimentos cognitivos mostram que as abelhas são capazes de discriminar compostos voláteis e até mesmo misturas de compostos que variam em proporções ou identidades de um ou mais componentes (Raguso 2008). Não existe, ainda, um modelo de percepção de fragrâncias como os existentes para percepção de cores.

\section{O PAPEL DAS PREFERÊNCIAS INATAS NA ESCOLHA DOS ESTÍMULOS}

As abelhas, assim como outros animais, possuem preferências por certos estímulos de maneira inata (Giurfa et al. 1995). Essas preferências foram descobertas em experimentos com abelhas inexperientes (também chamadas de naïve), ou seja, que nunca tiveram contato com os estímulos testados. Pela dificuldade de se isolar estímulos olfativos, uma vez que a própria colônia é um ambiente de exposição a diversos odores (Farina $e t$ $a l$.2005), incluindo feromônios que podem compor os voláteis das fragrâncias florais (Maisonnasse $e t$ al. 2010), experimentos de preferência inata são realizados com estímulos visuais. Por exemplo, sabe-se que A. mellifera possui preferência inata por flores azul-ultravioletas e verdes (Giurfa et al. 1995). De forma semelhante, descobriu-se que Bombus terrestris (Hymenoptera, Apidae) possui preferência inata por estímulos bilateralmente simétricos (Rodríguez et al. 2004). As preferências inatas podem ser suprimidas pelo aprendizado, mas em alguns casos elas ainda podem influenciar as escolhas de abelhas experientes, isto é, que já passaram por algum processo de treinamento associativo (Gumbert 2000).

\section{O PAPEL DO APRENDIZADO E AS BASES DO CONDICIONAMENTO}

Existem diferentes protocolos de condicionamento de abelhas. Nesta revisão, focaremos apenas no condicionamento apetitivo, pois a interação florpolinizador geralmente se dá por meio da busca de alimento pelos visitantes florais.

O aprendizado associativo é a capacidade dos animais extrair e associar informações do ambiente, estabelecendo uma relação de predição (Giurfa 2007). Isso difere da preferência inata, na qual não existe um aprendizado associativo e, portanto, não se estabelece uma predição. Por exemplo, o aprendizado associativo seria a capacidade cognitiva dos polinizadores associarem um atrativo floral (e.g., cor) à presença de néctar e então prever que a cor representa o recurso desejado. Existem duas formas de testar o aprendizado associativo: 1) condicionamento clássico (Pavlov 1927), no qual o animal aprende a associar um determinado sinal (i.e., estímulo condicionado, EC) com uma recompensa (i.e., estímulo não condicionado, ENC), a partir de uma resposta inata; e 2) condicionamento operante (Skinner 1938), em que a recompensa só é disponibilizada ao animal após uma mudança em seu comportamento. Essas duas formas de condicionamento podem ser atingidas em dois tipos de abordagens: o condicionamento absoluto, no qual o animal é treinado com apenas um estímulo condicionado (EC+) seguido de recompensa (ENC); e o condicionamento diferencial, no qual o animal é apresentado a dois estímulos, um associado a uma recompensa (EC+) e um sem a recompensa ou com uma punição, como solução de quinina ou sal que são substâncias de sabor aversivo para as abelhas (EC-). A apresentação de punição ao animal pode melhorar o processo 
de discriminação entre estímulos, pois os custos de uma resposta errada são maiores (Sanchez et al. 2015). Assim, no condicionamento diferencial, o animal deve associar um sinal à recompensa enquanto o outro sinal está associado a ausência de recompensa ou punição.

Qualquer experimento básico de aprendizado e memória em abelhas (seja clássico ou operante; absoluto ou diferencial) consiste em duas fases. $\mathrm{Na}$ primeira, denominada fase de aquisição ou treinamento, o animal é apresentado ao EC que é reforçado pelo ENC. A duração desta fase dependerá do estímulo (i.e., conspicuidade, atratividade) e/ou recompensa (i.e., quantidade, qualidade) (Menzel et al. 2001). Na segunda fase, a de teste, a abelha é novamente apresentada ao EC, mas também a outros cenários possíveis, dependendo do experimento. Em um deles, o EC estará desassociado do ENC. Nessa fase, a retirada da recompensa é importante para eliminar possíveis pistas químicas (i.e., odor do néctar, marcas deixadas pela própria abelha), restringindo a avaliação apenas entre as variáveis de interesse durante o treinamento (Matsumoto et al. 2012). Em outro cenário, um novo estímulo pode ser apresentado em conjunto ao EC, para estudar as capacidades de discriminação, quando o animal reconhece estímulos diferentes, ou generalização, quando o animal trata como equivalentes estímulos distintos (Guerrieri et al. 2005, Aguiar et al. 2018). Ainda, pode-se remover do teste o EC no qual a abelha foi originalmente treinada e apresentar um novo estímulo, para avaliar a transferência e a flexibilidade cognitiva das abelhas (Giurfa et al. 2001). No caso da polinização, as abelhas podem usar estímulos aprendidos em uma flor e transferir esse aprendizado para outras flores semelhantes no ambiente natural e, por isso, esses experimentos cognitivos podem trazer resultados interessantes nesse contexto.

Diversos materiais podem ser usados como ECs. Em experimentos de visão, podem ser utilizados desde papéis coloridos (Giurfa et al. 2001, Blackawton et al. 2011) até fontes de luz monocromáticas (Vieira et al. 2018). Em experimentos de olfação, geralmente são utilizados compostos comerciais puros (Matsumoto et al. 2012), ou então extratos de fragrância floral obtidos por cromatografia gasosa acoplada à espectrometria de massa (Locatelli et al. 2016). Já o ENC em condicionamento apetitivo deve ser um estímulo biologicamente relevante, que promova uma resposta inata de alimentação à abelha, sendo geralmente utilizada a solução de sacarose $50 \%$ (Matsumoto et al.2012). Estudos recentes também têm utilizado pólen como ENC (Nicholls \& Ibarra 2016). É importante ressaltar que a utilização de solução de sacarose como ENC em concentrações baixas $(<15 \%)$ pode atrapalhar a motivação apetitiva das abelhas, assim como soluções muito concentradas ( $>60 \%$ ), aumentando a viscosidade da solução e dificultando a ingestão (Matsumoto et al. 2012).

\section{COMO ACESSAR APRENDIZADO E MEMÓRIA NAS ABELHAS}

As abelhas podem ser treinadas individualmente em diversos desenhos experimentais. Isto é importante, pois aprendizado e memória se dão a partir da experiência do indivíduo (Giurfa 2007). Outra premissa importante, principalmente nos experimentos com abelhas imobilizadas, é a restrição alimentar. Controlar a alimentação das abelhas antes do treinamento influencia na motivação apetitiva durante o experimento (Matsumoto et al. 2012). Além disso, abelhas sociais possuem diferentes castas e, para estudos de polinização, comumente se usam apenas abelhas forrageiras, que de fato exploram as flores em busca de alimento.

Existe uma diversidade de variáveis resposta que podem ser mensuradas nos diferentes tipos de experimentos. Abelhas podem responder ao EC por aproximação ou pouso, que são as medidas mais comuns (Giurfa et al. 2001), ou então podese medir a antenação, quando a abelha toca o EC com as antenas antes de pousar, que consiste em uma forma de reconhecimento do estímulo nestes insetos (Lunau et al. 2006). Ainda, há a vibração do tórax, relacionada à comunicação sobre a qualidade de alimento às outras abelhas da colônia (Hrncir et al. 2004), e a extensão da probóscide, que indica resposta apetitiva (Matsumoto et al. 2012). A seguir explicaremos as bases de dois protocolos de condicionamento apetitivo que são úteis em questões de polinização.

\section{Resposta da extensão de probóscide}

Quando se toca a antena de uma abelha faminta 
com solução de sacarose, ela imediatamente estende a probóscide para se alimentar do açúcar (Giurfa 2007; Figura 1a). Baseado neste tipo de resposta, Takeda (1961)desenvolveu um protocolo de condicionamento clássico, denominado Resposta da Extensão de Probóscide (REP; Proboscis Extension Response). Nesse protocolo, a abelha associa um determinado estímulo com a disponibilidade de alimento. Este protocolo é mais recomendado para condicionamento olfativo, pois os estímulos químicos funcionam de maneira excelente. No entanto, com estímulos visuais, a resposta das abelhas não é tão eficiente nesse protocolo (Avarguès-Weber \& Mota 2016). O protocolo ainda permite investigar as bases neurais do aprendizado e da memória, pois é possível o acesso ao cérebro do animal simultaneamente à apresentação do estímulo (Rath et al. 2011). Usando como exemplo o condicionamento olfativo, um odorante neutro apresentado sem a recompensa não desencadeia o reflexo da extensão de probóscide. No entanto, se oodorante é apresentado antes da disponibilização da recompensa, uma associação é formada entre odorante (i.e., estímulo condicionado, EC) e a solução de sacarose (i.e., estímulo não condicionado, ENC). Isso permite que o odorante sozinho possa desencadear a REP em um teste subsequente ao treinamento (Giurfa 2007, Matsumoto et al. 2012).

Para o bom funcionamento desse protocolo, as abelhas devem ser imobilizadas (Figura 1a), de forma que possam mover apenas as antenas e as peças bucais (Matsumoto et al. 2012). As abelhas imobilizadas devem ser mantidas sem alimentação de duas a três horas antes do início do condicionamento para assegurar motivação apetitiva. Nesse período a abelha deve ser mantida
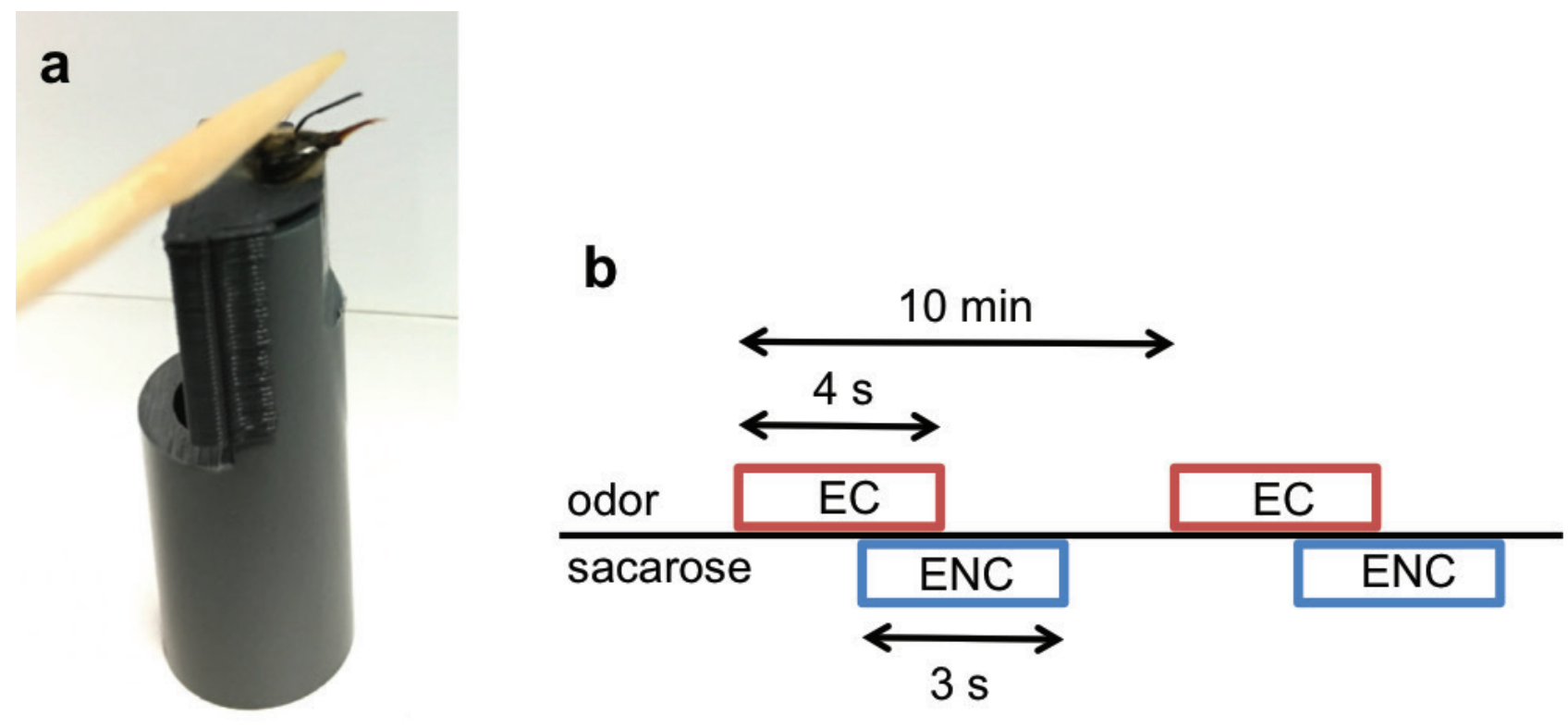

Figura 1. Resposta da extensão de probóscide (REP). a) Indivíduo de Apis melífera (Hymenoptera, Apidae) imobilizado em tubo de plástico. Na figura vemos um palito de dente embebido em solução de sacarose tocando a antena da abelha, o que desencadeia a resposta inata da extensão de probóscide. b) Esquema básico do condicionamento no protocolo REP baseado em Matsumoto et al. (2012). O condicionamento consiste no pareamento de quatro segundos de apresentação do estímulo condicionado (EC; odor; retângulos vermelhos) e a subsequente apresentação do estímulo não condicionado por três segundos (ENC; sacarose; retângulos azuis), com um segundo de sobreposição. Após dez minutos o mesmo indivíduo passa por um novo pareamento, e o processo pode ser repetido por quantas vezes o pesquisador achar necessário antes da fase de teste.

Figure 1. Proboscis extension response (PER). a) Individual of Apis mellifera (Hymenoptera, Apidae) harnessed in a plastic tube. In the figure we see a toothpick with sucrose solution touching the bee's antenna, which triggers the innate response of proboscis extension. b) Basic scheme of the PER conditioning protocol procedure based on Matsumoto et al. (2012). Conditioning consists in the pairing of four seconds of stimulus presentation (EC; odor; red rectangles) and the subsequent presentation of the unconditioned stimulus for three seconds (ENC; sucrose; blue rectangles), with one second overlap. After ten minutes, the same individual goes through a new pairing, and the process can be repeated as many times the researcher believes it is necessary before the test phase. 
em um local escuro e úmido, com temperatura controlada (Matsumoto et al. 2012). Para controle, deve-se confirmar que o estímulo é neutro à abelha, ao comprovar ausência de respostas ao EC antes do condicionamento. Também devese quantificar as respostas ao ENC antes e após o experimento, para assegurar que o animal possui a resposta não condicionada mesmo após a manipulação. Abelhas que não apresentem REP durante o teste controle ao ENC devem ser removidas do experimento, uma vez que podem gerar falsos negativos (Giurfa 2007).

No protocolo básico de condicionamento absoluto de REP, a fase de aquisição ocorre da seguinte forma: a abelha imobilizada é mantida por 25 seg para adaptação ao local do condicionamento, posicionada frente a um exaustor que impede a acumulação de odores, no caso tanto EC quanto feromônios liberados pelo animal. Após esse período, é apresentado o EC ao animal por quatro segundos com a subsequente apresentação do ENC por três segundos, com um segundo de sobreposição (Figura 1b). Após o pareamento EC-ENC a abelha é mantida por mais 25 seg no local do condicionamento. Para um condicionamento de múltiplas repetições, é recomendado um intervalo entre repetições de dez minutos (Matsumoto et al. 2012). O tempo de cada etapa é ajustável dependendo das condições do laboratório, das perguntas em questão e do modelo animal escolhido.

$\mathrm{Na}$ fase de teste, o intervalo de duração após o condicionamento depende da pergunta, que pode testar memórias de curto prazo (i.e., imediatamente após o condicionamento), médio prazo (i.e., após uma hora do condicionamento, por exemplo) ou longo prazo (i.e., até 72 horas após o condicionamento) (Matsumoto et al. 2012). Para testes após longos intervalos, o investigador deve alimentar as abelhas para evitar a morte por inanição. O teste deve ser feito com o EC e ao menos mais um estímulo novo não condicionado anteriormente, apresentados separadamente, funcionando como um controle para o caso de a abelha aprender a responder apenas ao EC (Matsumoto et al. 2012). Respostas ao estímulo novo podem, no entanto, fornecer evidências sobre generalização (Guerrieri et al. 2005).

Para condicionamento diferencial de REP, os EC+ e EC- devem ser apresentados um de cada vez, com a mesma quantidade de repetições, de forma randomizada. O EC-pode ser a ausência de solução de sacarose, ou ainda, substâncias aversivas para as abelhas. Recomenda-se solução concentrada de cloreto de sódio $(\mathrm{NaCl})$ nesses casos (Sanchez et al. 2015). A apresentação de EC às abelhas, no caso do condicionamento olfativo, pode ser feita de forma precisa, com aparelhos controlados por microcomputadores (Szyszka et al. 2014), o que permite um melhor controle experimental. Porém, resultados confiáveis também são obtidos de forma mais simples, utilizando seringas contendo um pedaço de papel filtro embebido em odorante (Matsumoto et al. 2012).

\section{Abelhas em voo livre}

Apesar do protocolo de REP mostrar resultados consistentes e robustos (Matsumoto et al. 2012), argumenta-se que ele pode limitar as respostas comportamentais das abelhas, uma vez que o animal está imobilizado. Numa tentativa de contornar tal problema, experimentos com abelhas em voo livre podem ser realizados. No entanto, testes com fragrâncias são difíceis de serem apresentados nesse protocolo, pois se misturam no ambiente experimental. Além disso, experimentos em voo livre também possuem complicações, como a duração, podendo uma seção experimental ser bem mais longa em comparação ao protocolo de REP.

O experimento de voo livre é considerado essencialmente um condicionamento operante, já que a abelha deve alterar seu comportamento (i.e., visitar o estímulo correto) para obter a recompensa. No entanto, a associação entre EC e ENC nesse caso é considerada um misto de clássico e operante, pois a abelha pode vincular o EC diretamente ao ENC (condicionamento clássico) e simultaneamente modificar seu comportamento de escolha entre diferentes estímulos ao ENC (condicionamento operante) (Giurfa 2007).

Para o condicionamento em voo livre o treinamento pode ter início de diversas formas como, por exemplo, com um alimentador contendo solução de sacarose posicionado próximo à colônia (Figura 2a). À medida que as abelhas visitem o alimentador, deve-se afastá-lo pouco a pouco até o local do experimento (Roselino et al. 2016). O alimentador não pode conter estímulos semelhantes aos que serão testados, e 

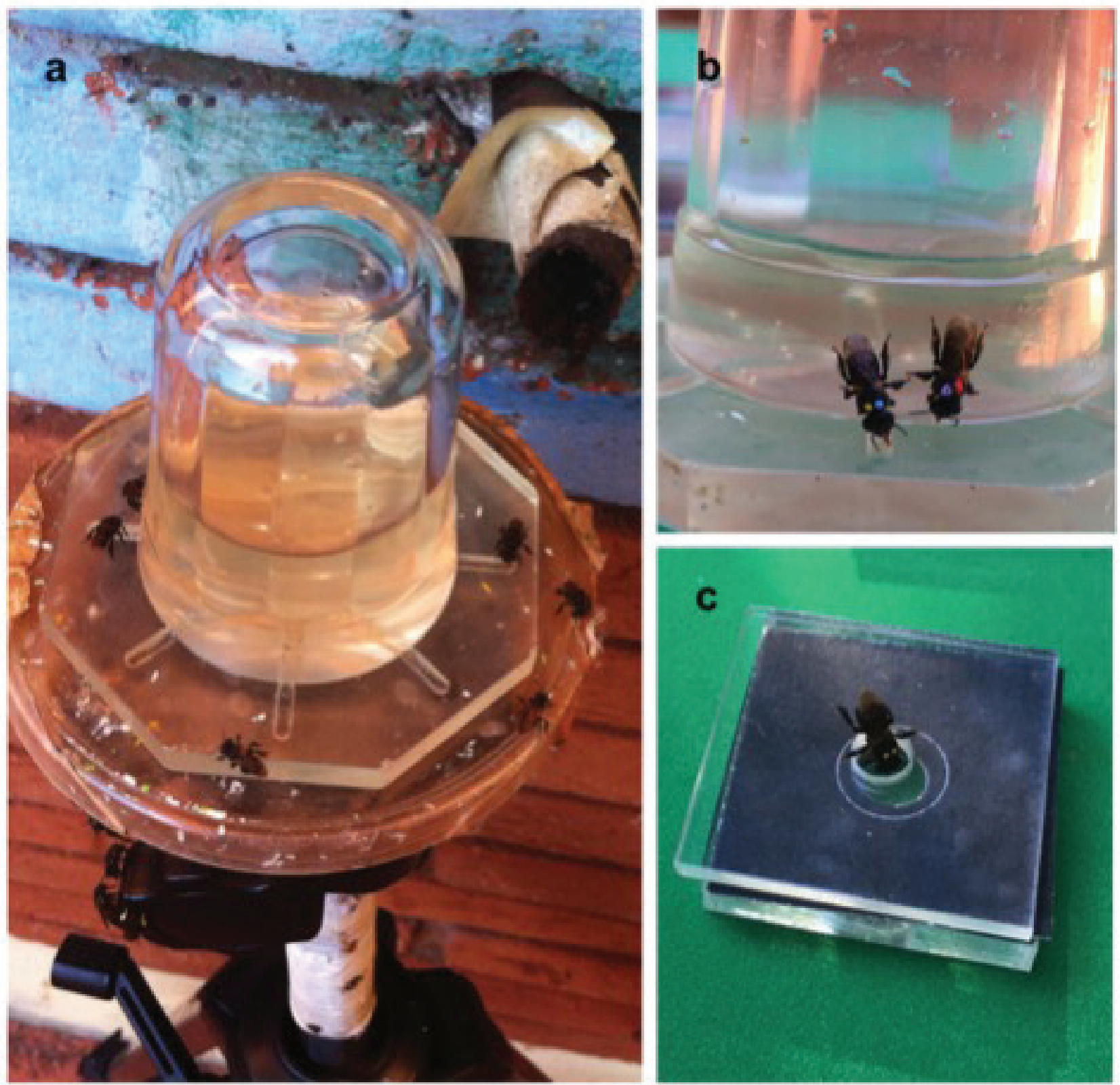

Figura 2. Treinamento de abelhas em voo livre. a) Alimentador de gravidade posicionado em frente à entrada de uma colônia de Scaptotrigona aff. depilis (Hymenoptera, Apidae). O alimentador consiste em um copo de vidro com solução de sacarose, sobre uma placa de acrílico com vincos por onde as abelhas podem acessar o alimento. b) Abelhas, marcadas no tórax com tinta acrílica, no alimentador de gravidade. c) Exemplo de uma flor artificial que pode ser utilizada em condicionamentos em voo livre. A flor consiste em uma base de acrílico com uma cavidade na qual é colocada a solução de sacarose. Sobre essa base, é posicionado o estímulo, no caso um quadrado de papel cinza, e sobre o estímulo é posicionada uma lâmina de acrílico perfurada. A lâmina deve ser trocada a cada visita e limpa após o experimento para evitar a influência de possíveis marcas de odor (feromônios) deixadas pela abelha.

Figure 2. Free-flight bee training. a) Gravity feeder placed in front of a nest of Scaptotrigona aff. depilis (Hymenoptera, Apidae). The feeder consists in a glass cup with sucrose solution over a Plexiglas plate with incisions where bees can access the food. b) Bees marked with acrylic paint at the thorax visiting the gravity feeder. c) Example of artificial flower that can be used in free-flight conditioning. The flower consists in a Plexiglas base with a cavity in which the sucrose solution is placed. Over this base, the stimulus is placed (in the example, a gray paper square). A Plexiglas sheet is placed over the stimulus. This sheet must be replaced after each visit and properly cleaned after the experiments to avoid the influence of possible odor cues (pheromones) by the bees. 
de preferência, neutro quanto à sua coloração (i.e., transparente). Sugere-se utilizar copos de vidro com solução de sacarose entornado sobre uma placa de acrílico com vincos, permitindo a disponibilização gradual da solução (Figura 2a). O intervalo entre repetições dependerá da distância do local do experimento até a entrada da colônia. Para uma distância de 100 m, abelhas motivadas demoram de dois a dez minutos entre viagens (Giurfa 2007). Cada abelha deve ser individualizada, por exemplo, através de marcações com tinta atóxica no tórax (Figura 2b), ou tags numeradas aderidas ao tórax.

Pode-se realizar tanto condicionamento absoluto como diferencial com abelhas em voo livre. No entanto, diferentemente do protocolo de REP, ambos EC+ e EC- são apresentados simultaneamente no teste. Já o número de repetições é relativo ao número de viagens da abelha, e não mais ao número de apresentações do EC como para o condicionamento de REP. Sugerese dez repetições de treinamento, com a fase teste sendo realizada logo após o condicionamento. Diversos comportamentos podem ser utilizados como variável resposta, como antenação ou pouso do animal (Giurfa 2007).

A instalação experimental é variável, se ajustando ao desenho experimental (Lehrer et al. 1995, Stach \& Giurfa 2005). Aqui explicaremos duas formas de condicionamento visual existentes. A primeira consiste na utilização de flores artificiais. Uma flor artificial pode ser construída em base de acrílico com cavidade que permita o oferecimento de solução de sacarose. Sobre a base, se posiciona um papel colorido furado e sobre o papel, uma lâmina de acrílico perfurada (Roselino et al. 2016; Figura 2c). O propósito dessa lâmina de acrílico é evitar o acúmulo de marcas de feromônio. Assim, tal lâmina deve ser trocada após cada visita e toda a estrutura de acrílico da flor artificial limpa com álcool após o experimento (Roselino et al. 2016).

Outra instalação experimental é o labirinto emY (Figura 3). Nesse caso, a abelha é treinada a entrar em uma câmara de dois braços unidos por um corredor. O material para confecção varia, podendo ser acrílico ou madeira. No corredor existe uma abertura à frente de uma porta deslizante, que é fechada após a passagem da abelha, permitindo o treinamento individual (Giurfa et al. 2001, Stach \& Giurfa 2005). Geralmente, em apenas um dos braços está o estímulo com recompensa, enquanto no outro braço, outro estímulo sem recompensa é apresentado (condicionamento diferencial). Ainda, para evitar o uso de pistas químicas provenientes do néctar ou pólen, na fase de teste, os dois estímulos (de cada braço) são apresentados sem recurso na fase teste. O labirinto em Y permite controlar a distância do estímulo ao ponto de decisão da abelha, que é o encontro do corredor com os dois

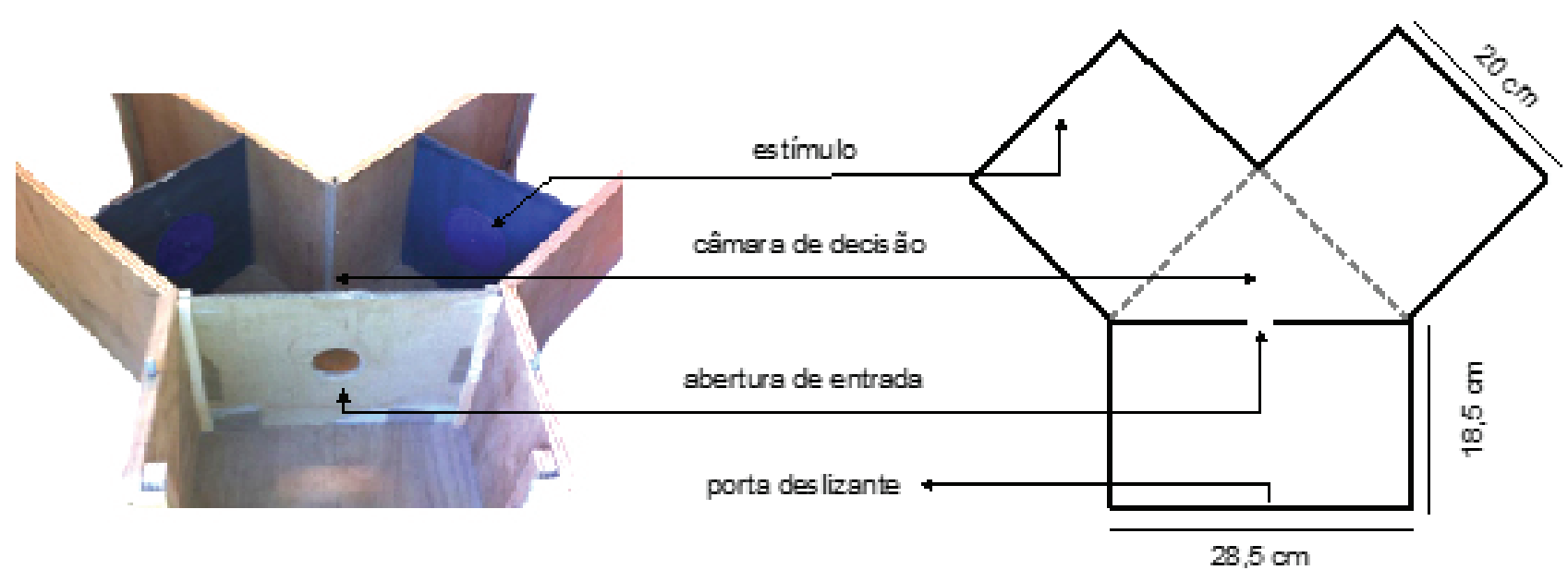

Figura 3. Exemplo de um labirinto em Y para condicionamento em voo livre. A abelha pode ser treinada a entrar no labirinto no qual cada braço apresenta um estímulo na parede ao fundo. Geralmente, apenas um dos estímulos recebe a solução de sacarose. A distância entre os estímulos e a câmara de decisão pode variar, para avaliar o efeito do ângulo de visão do polinizador no condicionamento. Foto: Paul Marchal. Dimensões baseadas em Stach \& Giurfa (2005).

Figure 3. Example of a Y-maze for free-flight conditioning. The bee can be trained to enter the maze, where each arm presents a stimulus at the back wall. Usually, only one of the stimuli contains sucrose solution. The distance between stimuli and the decision chamber can vary, to evaluate the effect of the pollinator's visual angle in conditioning. Photo: Paul Marchal. Dimentions based on Stach \& Giurfa (2005). 
braços, onde a abelha enxerga simultaneamente os dois estímulos (Giurfa et al. 1996). A um ângulo de visão menor (i.e., maior distância do animal ao estímulo), as abelhas utilizam apenas o fotorreceptor verde para identificar um objeto. Já a uma distância menor, todos os fotorreceptores são ativados e as abelhas utilizam visão em cor para identificar um objeto (Giurfa et al. 1996). Essa relação tem implicações diretas em biologia da polinização como será exposto na próxima seção.

\section{APLICAÇÕES NOS ESTUDOS DE BIOLOGIA DA POLINIZAÇÃO}

\section{Isômeros das fragrâncias florais}

A fragrância floral inclui vários compostos voláteis, com variação até entre indivíduos de uma população. Por exemplo, muitas orquídeas polinizadas por engano de alimento apresentam variação intraespecífica destes compostos. Parte dessa variação é devida à presença de isômeros (Salzmann et al.2007, Dormont et al. 2014), ou seja, diferentes conformações estruturais ou espaciais de uma mesma molécula (IUPAC, http:/ /goldbook. iupac.org). Esta variação intraespecífica em flores polinizadas por engano seria uma estratégia adaptativa, dificultando aos polinizadores aprenderem a associar os estímulos florais com a ausência de recursos quando a informação atrativa é altamente variável (Heinrich 1975). Assim, os polinizadores demorariam mais para aprender a evitar essas flores, pois teriam que associar todos os estímulos possíveis à ausência de recurso. Por consequência, estas flores teriam maior taxa de visitação quando comparadas a outras flores de engano sem tal variação. Dessa forma, diferentes isômeros na fragrância floral poderiam atuar nessa interferência no aprendizado dos polinizadores. A pergunta primordial é saber se os polinizadores conseguem diferenciar tais isômeros. Essa foi a pergunta central do estudo de Aguiar et al. (2018).

Investigando a fragrância floral de Ionopsis utricularioides (Asparagales, Orchidaceae), uma orquídea polinizada por engano de alimento, Aguiar \& Pansarin (2019), encontraram grande variação intraespecífica oriunda, também, da presença de isômeros. Assim, selecionaram quatro pares de isômeros presentes nas fragrâncias de orquídeas polinizadas por engano de alimento, incluindo I. utricularioides. Utilizando o protocolo de REP, testaram a capacidade de discriminação entre essas moléculas, que atuaram como EC, por A. mellifera. Neste caso, o ENC foi a ausência de solução de sacarose ou solução concentrada de NaCL. Em condicionamento absoluto as abelhas não conseguiram diferenciar a maioria dos isômeros. Porém, em condicionamento diferencial, as abelhas diferenciaram alguns pares de isômeros. Apesar disso, a taxa de generalização entre estímulos foi muito alta, ou seja, mesmo quando há diferenciação, as abelhas ainda respondem muito ao isômero treinado como EC-.

Então, as abelhas conseguem discriminar pares de isômeros em algumas situações experimentais específicas, mas a tendência é ocorrer generalização entre essas moléculas (Aguiar et al. 2018). Num contexto de polinização em condições naturais, seria difícil para uma abelha discriminar isômeros e utilizá-los como preditores da ausência de recurso. Como conclusão, os isômeros não contribuiriam para a variabilidade da fragrância floral que pode ser percebida pelos polinizadores.

\section{Mudança de cor floral}

Em muitas plantas ocorre mudança de cor da flor ao longo da antese associada a manutenção de flores velhas. Estas flores velhas geralmente estão desprovidas de recurso e não são visitadas pelos polinizadores (Weiss 1991, Weiss \& Lamont 1997). Existem duas principais hipóteses não excludentes e associadas para explicar tal fenômeno (Weiss 1991): a hipótese de longa distância diz que a mudança de cor floral e manutenção de flores velhas torna o display floral maior e mais atrativo visto de longe. Já a hipótese de curta distância diz que, vistas de perto, as diferentes cores direcionam os polinizadores para as flores novas, diminuindo as visitas em flores velhas e não receptivas e otimizando a polinização. Para corroborar estas duas hipóteses, as diferentes cores não devem ser discriminadas de longe, pois as abelhas poderiam prever que o maior display não significa maior oferta de recurso, ao mesmo tempo que devem ser discriminadas de perto.

Essa hipótese foi testada para Tibouchina pulchra (Myrtales, Melastomataceae), uma planta que muda a cor floral de branco para rosa, utilizando-se modelos visuais e experimentos em laboratório (Brito et al. 2015). Os modelos mostraram que, independentemente da cor, as 
flores possuem alta saturação, um parâmetro importante para escolha a longas distâncias. Já a curtas distâncias elas são discrimináveis pelas abelhas. Em laboratório, utilizando-se labirinto em Y, demostrou-se que à longas distâncias as abelhas naïve preferem árvores artificiais com retenção de flores velhas que árvores sem retenção de flores velhas. Situação semelhante ocorre a curtas distâncias: as abelhas preferem um display de flores com cores diferentes que flores com cores iguais, mas com variação intrafloral na cor. Assim, corroboraram-se as hipóteses de longa e curta distâncias para explicar um fenômeno que ocorre em mais de 250 gêneros de plantas (Weiss \& Lamont 1997).

\section{Estímulos complexos}

As flores geralmente apresentam atrativos complexos, seja por meio de sinais multimodais (e.g., cor e odor) ou multicomponente (e.g., formato, tamanho e cor) (Melo et al. 2018). Porém, o forrageamento das abelhas quando na presença de atrativos complexos é pouco entendido. Será que determinadas combinações (e.g., estímulo visualmente saliente + odor; estímulo menos saliente + odor, e assim por diante) são redundantes ou têm efeito aditivo sobre os visitantes florais?

A maioria dos estudos se concentra apenas em modalidades sensoriais simples para compreender o papel da cognição dos polinizadores e prever o comportamento de forrageio. Mas, para entender como as abelhas lidam com situações de complexidade do sinal, o papel de sinais florais complexos deve se tornar parte do desenho experimental. Este foi um dos objetivos do estudo de Telles et al. (2017). Os pesquisadores exploraram o papel de sinais florais complexos, multimodais (i.e., cor + odor) e multicomponentes (i.e., cor + tamanho), no forrageamento de um polinizador generalista (B. terrestris). Para tal, utilizaram protocolo de voo livre: as abelhas eram condicionadas a forragear em uma caixa de voo (5 $\mathrm{m} \times 2,5 \mathrm{~m}$ ) em condições seminaturais, no que se refere à iluminação e a complexidade do plano de fundo onde os estímulos eram apresentados.

De todos os estímulos considerados no estudo, a cor foi o que influenciou fortemente o comportamento de forrageamento das abelhas (Telles et al. 2017). Apesar da ideia de que o sistema sensorial dos polinizadores e os sinais florais funcionam sinergicamente para aumentar a detecção de estímulos e a constância de visitas, os indivíduos testados no estudo nem sempre fizeram uso dos sinais complexos. O desempenho das abelhas procurando por flores menos conspícuas (i.e., vermelhas UV-absorventes) melhorou na presença de odor, enquanto a frequência de visitas a flores conspícuas (i.e., azul) na presença de odor não diferiu do tratamento usando as mesmas flores na ausência de odor. Assim, quando o estimulo é visualmente saliente, a presença de um segundo sinal sensorial (i.e., odor) torna-se irrelevante (Telles et al. 2017).

\section{NOVAS ABORDAGENS}

Ainda que abordagens clássicas de condicionamento sejam eficientes para entender os processos cognitivos das abelhas, novas propostas têm aprimorado os experimentos. Uma das dificuldades para realizar o protocolo de resposta de extensão de probóscide (REP) é a aparelhagem necessária em laboratório, a qual é dispendiosa e ocupa espaço. Além disso, a necessidade de manter as abelhas em colônias reduz a diversidade de espécies que podem ser utilizadas. Muth etal.(2018) propõem um novo condicionamento visual de REP de baixo custo em campo com abelhas selvagens. Nesse trabalho, abelhas coletadas em seu ambiente natural foram posicionadas individualmente dentro de um tubo de acrílico, onde podiam caminhar livremente. No tubo foi inserida uma tira de papel colorida (EC) embebida em solução de sacarose (ENC), e a abelha podia dirigir-se até a tira e estender a probóscide para provar a recompensa. Em condicionamento absoluto a abelha foi apresentada a cinco repetições e cinco minutos após a última repetição foram inseridas duas tiras de cores diferentes sem ENC, sendo uma das cores o EC. Pode ser realizado também condicionamento diferencial em que EC+ e EC- são apresentados em repetições separadas. As abelhas escolheram preferencialmente o EC, compatível com resultados obtidos com o protocolo clássico de REP.

Em condicionamento de voo livre, as abelhas conseguem realizar tarefas complexas de discriminação de estímulos visuais, porém não é possível explorar as bases neurofisiológicas como se faz em abelhas imobilizadas (Avarguès-Weber \& Mota 2016). No entanto, abelhas imobilizadas 
não possuem boa performance de discriminação visual no protocolo clássico de REP (AvarguèsWeber \& Mota 2016). Buscando superar estas limitações, Buatois et al.(2017) desenvolveram um equipamento e protocolo de condicionamento visual em que a abelha fica imersa em uma realidade virtual. Nesse protocolo, a abelha fica presa por um fio aderido ao seu tórax e é posicionada sobre uma esfera de isopor suspensa por um fluxo de ar. A abelha pode andar livremente rolando a esfera e esse movimento é capturado por sensores conectados a um equipamento que projeta os EC+ e EC- sobre uma tela. Conforme a abelha anda sobre a esfera, os sensores atualizam a posição dos estímulos na tela, criando um ambiente imersivo. Se as abelhas andam em direção ao EC+ até que ele fique no centro da tela, elas recebem a solução de sacarose. Os resultados foram semelhantes aos obtidos utilizando labirinto em Y (Buatois et al. 2018), com a vantagem que abelhas parcialmente imobilizadas podem ser utilizadas em análises neurofisiológicas.

\section{CONCLUSÃO}

As abelhas são capazes de realizar tarefas complexas, importantes no reconhecimento e aprendizado de fontes de alimento em seu ambiente natural. Devido às facilidades de treinamento, foi possível desenvolver diversos protocolos e equipamentos para o estudo das capacidades cognitivas importantes para o processo de polinização. Cada protocolo tem suas vantagens e desvantagens, e estão sendo constantemente aprimorados com o surgimento de novas tecnologias e informações a respeito da cognição das abelhas. Nesta revisão, buscamos trazer conceitos básicos dos estudos cognitivos usando esses insetos como modelos, como uma primeira leitura para entrar em contato com esse campo em plena expansão. Quando tratamos de biologia da polinização, é importante ter em mente que os polinizadores não percebem as flores como os humanos. Assim, os experimentos cognitivos são a melhor maneira de entender como um polinizador percebe, aprende, memoriza e usa essa informação para tomar suas decisões. Dessa forma, é importante que o pesquisador aprenda a treinar a sua abelha e, portanto, entenda o processo de polinização pelos "olhos e narizes" do polinizador.

\section{AGRADECIMENTOS}

À Fundação de Apoio à Pesquisa do Estado de São Paulo (FAPESP), pela bolsa de Doutorado concedida à JMRBVA (processo 2015/05919-8) e à PJJB (processo 2016/06434-0). À Fundação de Apoio à Pesquisa do Estado de Minas Gerais (FAPEMIG) pelo auxílio à pesquisa a VLGB (processo APQ02497-16). À Coordenação de Aperfeiçoamento de Pessoal de Nível Superior - Brasil (CAPES) Código de Financiamento 001, e pela bolsa PNPD (CAPES-1659767) concedida à FJTs. MS agradece ao Conselho Nacional de Desenvolvimento Científico e Tecnológico (CNPq, processo 302781/2016-1). À Paul Marchal (CRCA, Université Paul Sabatier Toulouse III) por gentilmente ceder a foto da Figura 3. Ao trabalho de revisão realizado por Carlos E. P. Nunes, Leandro S. L. Hohl e um terceiro revisor anônimo.

\section{REFERÊNCIAS}

Aguiar, J. M. R. B. V., Roselino, A. C., Sazima, M., \& Giurfa, M. 2018. Can honey bees discriminate between floral-fragrance isomers? Journal of Experimental Biology, 221(14), jeb180844. DOI: $10.1242 /$ jeb. 180844

Aguiar, J. M. R. B. V., \& Pansarin, E. R. 2019. Deceptive pollination of Ionopsis utricularioides (Oncidiinae: Orchidaceae). Flora: Morphology, Distribution, Functional Ecology of Plants, 250(1), 72-78. DOI: 10.1016/j.flora.2018.11.018

Avarguès-Weber, A., \& Mota, T. 2016. Advances and limitations of visual conditioning protocols in harnessed bees. Journal of Physiology Paris, 110(3), 107-118. DOI: 10.1016/j. jphysparis.2016.12.006

Balamurali, G. S., Nicholls, E., Somanathan, H., \& Ibarra, N. H. 2018. A comparative analysis of colour preferences in temperate and tropical social bees. The Science of Nature, 105(1-2), 1-8. DOI: $10.1007 / \mathrm{s} 00114-017-1531-z$

Baldelomar, M., Viana, M. L., \& Telles, F. J. 2018. El rol de los compuestos orgánicos volátiles florales en las interacciones planta-insecto. Oecologia Australis, 22(4), 348-361. DOI: 10.4257/oeco.2018.2204.02

Bergamo, P. J., Telles, F. J., Arnold, S. E. J., \& Brito, V. L. G. 2018. Flower colour within communities shifts from overdispersed to clustered along an 
alpine altitudinal gradient. Oecologia, 188(1), 223-235. DOI: 10.1007/s00442-018-4204-5

Blackawton, P. S., Airzee, S., Allen, A., Baker, S., Berrow, A., Blair, C., Churchill, M., Coles, J., Cumming, R. F. J., Fraquelli, L., Hackford, C., Hinton Mellor, A., Hutchcroft, M., Ireland, B., Jewsbury, D., Littlejohns, A., Littlejohns, G., Lotto, M., McKeown, J., O’Toole, A., Richards, H., Robbins-Davey, L., Roblyn, S., Rodwell-Lyn, H., Schenck, D., Springer, J., WishyA., Rodwell-Lynn, T., Strudwick, D., \& Lotto, R. B. 2011. Blackawton bees. Biology Letters, 7(2), 168-172. DOI: 10.1098/ rsbl.2010.1056

Briscoe, A. D., \& Chittka, L. 2001. The evolution of color vision in insects. Annual Review of Entomology, 46(1), 471-510. DOI: 10.1146/ annurev.ento.46.1.471

Brito, V. L. G., Weynans, K., Sazima, M., \& Lunau, K. 2015. Trees as huge flowers and flowers as oversized floral guides: the role of floral color change and retention of old flowers in Tibouchina pulchra. Frontiers in Plant Science, 6(1), 1-10. DOI: 10.3389/fpls.2015.00362

Brito, V. L. G., Telles, F. J., \& Lunau, K. 2014. Ecologia cognitiva da polinização. In: A. R. Rech, K. Agostini, P. E. Oliveira, \& I. C. Machado (Eds.), Biologia da polinização. pp. 417-438. Rio de Janeiro: Projeto Cultural.

Buatois, A., Flumian, C., Schultheiss, P., AvarguèsWeber, A., \& Giurfa, M. 2018. Transfer of visual learning between a virtual and a real environment in honey bees: the role of active vision. Frontiers in Behavioral Neuroscience, 12(1), 1-17. DOI: 10.3389/fnbeh.2018.00139

Buatois, A., Pichot, C., Schultheiss, P., Sandoz, J. C., Lazzari, C. R., Chittka, L., Avarguès-Weber, A., \& Giurfa, M. 2017. Associative visual learning by tethered bees in a controlled visual environment. Scientific Reports, 7(1), 12903. DOI: 10.1038/ s41598-017-12631-w

Chittka, L. 1997. Bee color vision is optimal for coding flower color, but flower colors are not optimal for being coded-why? Israel Journal of Plant Sciences, 45(2-3), 115-127. DOI: 10.1080/07929978.1997.10676678

Chittka, L., Faruq, S., Skorupski, P., \& Werner, A. 2014. Colour constancy in insects. Journal of Comparative Physiology A: Neuroethology, Sensory, Neural, and Behavioral Physiology, 200(6), 435-448. DOI: 10.1007/s00359-014-0897-z
Chittka, L., \& Thomson, J. D. 2004. Cognitive ecology of pollination: animal behavior and floral evolution. New York: Cambridge University Press: p. 344. DOI: 10.1046/j.14390310.2002.00774.x

Chittka, L., Thomson, J. D., \& Waser, N. M. 1999. Flower constancy, insect psychology, and plant evolution. Naturwissenschaften, 86(8), 361-377. DOI: $10.1007 / \mathrm{s} 001140050636$

Dormont, L., Delle-Vedove, R., Bessière, J. M., \& Schatz, B. 2014. Floral scent emitted by white and coloured morphs in orchids. Phytochemistry, 100(1), 51-59. DOI: 10.1016/j. phytochem.2014.01.009

Dyer, A. G., Garcia, J. E., Shrestha, M., \& Lunau, K. 2015. Seeing in colour: a hundred years of studies on bee vision since the work of the Nobel laureate Karl von Frisch. Proceedings of the Royal Society of Victoria, 127(1), 66. DOI: 10.1071/RS15006

Farina, W. M., Grüater, C., \& Díaz, P. C. 2005. Social learning of floral odours inside the honeybee hive. Proceedings of the Royal Society B: Biological Sciences, 272(1575), 1923-1928. DOI: 10.1098/rspb.2005.3172

Frisch, K. 1914. Der farbensinn und Formensinn der Biene. Zoologische Jahrbücher. Abteilung für Allgemeine Zoologie und Physiologie der Tiere: p. 182.

Gawryszewski, F. M. 2018. Color vision models: Some simulations, a general $\mathrm{n}$ - dimensional model, and the colourvision R package. Ecology and Evolution, 8(1) 8159-8170. DOI: 10.1002/ ece3.4288

Giurfa, M., Núñez, J., Chittka, L., \& Menzel, R. 1995. Colour preferences of flower-naive honeybees. Journal of Comparative Physiology A, 177(3), 247-259. DOI: 10.1007/BF00192415

Giurfa, M., Vorobyev, M., Kevan, P. G., \& Menzel, R. 1996. Detection of coloured stimuli by honeybees: minimum visual angles and receptor specific contrasts. Journal of Comparative Physiology A, 178(5), 699-709.

Giurfa, M. 2004. Conditioning procedure and color discrimination in the honeybee Apis mellifera. Naturwissenschaften, 91(5), 228-231. DOI: 10.1007/s00114-004-0530-z

Giurfa, M. 2007. Behavioral and neural analysis of associative learning in the honeybee: a taste from the magic well. Journal of Comparative 
Physiology A, 193(8), 801-24. DOI: 10.1007/ s00359-007-0235-9

Giurfa, M., Zhang, S., Jenett, A., Menzel, R., \& Srinivasan, M. V. 2001. The concepts of "sameness" and "difference" in an insect. Nature, 410(6831), 930-933. DOI: 10.1038/35073582

Guerrieri, F, Lachnit, H., Gerber, B., \& Giurfa, M. 2005. Olfactory blocking and odorant similarity in the honeybee. Learning and Memory, 12(2), 86-95. DOI: 10.1101/lm.79305

Gumbert, A. 2000. Color choices by bumble bees (Bombus terrestris): innate preferences and generalization after learning. Behavioral Ecology and Sociobiology, 48(1), 36-43. DOI: $10.1007 / \mathrm{s} 002650000213$

Heinrich, B. 1975. Bee flowers: a hypothesis on flower variety and blooming times. Evolution, 29(2), 325-334. DOI: 10.2307/2407220

Henske, J., Krausa, K., Hager, F. A., Nkoba, K., \& Kirchner, W. H. 2015. Olfactory associative learning in two African stingless bee species (Meliponula ferruginea and M. bocandei, Meliponini). Insectes Sociaux, 62(4), 507-516. DOI: $10.1007 / \mathrm{s} 00040-015-0430-6$

Hrncir, M., Jarau, S., Zucchi, R., \& Barth, F. G. 2004. Thorax vibrations of a stingless bee (Melipona seminigra). II. Dependence on sugar concentration. Journal of Comparative Physiology A: Neuroethology, Sensory, Neural, and Behavioral Physiology, 190(7), 549-560. DOI: 10.1007 / s00359-004-0515-6

Kemp, D. J., Herberstein, M. E., Fleishman, L. J., Endler, J. A., Bennett, A. T. D., Dyer, A. G., Hart, N. S., Marshall, J., \& Whiting, M. J. 2015. An integrative framework for the appraisal of coloration in nature. The American Naturalist, 185(6), 705-724. DOI: 10.1086/681021

Koethe, S., Bossems, J., Dyer, A. G., \& Lunau, K. 2016. Colour is more than hue: preferences for compiled colour traits in the stingless bees Melipona mondury and M. quadrifasciata. Journal of Comparative Physiology A: Neuroethology, Sensory, Neural, and Behavioral Physiology, 202(9-10), 615-627. DOI: 10.1007/ s00359-016-1115-y

Land, M. F., \& Nilsson, D. E. 2002. Animal eyes. London: Oxford University Press: p. 221.

Lehrer, M., Horridge, G. A., Zhang, S. W., \& Gadagkar, R. 1995. Shape vision in bees: innate preference for flower-like patterns. Philosophical
Transactions of the Royal Society B: Biological Sciences, 347(1320), 123-137. DOI: 10.1098/ rstb.1995.0017

Locatelli, F. F., Fernandez, P. C., \& Smith, B. H. 2016. Learning about natural variation of odor mixtures enhances categorization in early olfactory processing. Journal of Experimental Biology, 219(17), 2752-2762. DOI: 10.1242/ jeb.141465

Lunau, K., Fieselmann, G., Heuschen, B., \& Loo, A. 2006. Visual targeting of components of floral colour patterns in flower-naïve bumblebees (Bombus terrestris; Apidae). Naturwissenschaften, 93(7), 325-8. DOI: 10.1007/s00114-006-0105-2

Maisonnasse, A., Lenoir, J. C., Beslay, D., Crauser, D., \& Conte, Y. 2010. E- $\beta$-ocimene, a volatile brood pheromone involved in social regulation in the honey bee colony (Apis mellifera). PLoS ONE, 5(10), e13531. DOI: 10.1371/journal. pone. 0013531

Matsumoto, Y., Menzel, R., Sandoz, J. C., \& Giurfa, M. 2012. Revisiting olfactory classical conditioning of the proboscis extension response in honey bees: a step toward standardized procedures. Journal of Neuroscience Methods, 211(1), 159167. DOI: $10.1016 /$ j.jneumeth.2012.08.018

Melo, L. R. F., Guimarães, B. M. C., Barônio, G. J., Oliveira, L. C., Cardoso, R. K. O. A., Araújo, T. N., \& Telles, F. J. 2018. Como as abelhas percebem as flores e por que isto é importante? Oecologia Australis, 22(4), 362-389. DOI: 10.4257/ oeco.2018.2204.03

Menzel, R., Manz, G., Menzel, R., \& Greggers, U. 2001. Massed and spaced learning in honeybees: the role of CS, US, the intertrial interval, and the test interval. Learning and Memory, 8(4), 198208. DOI: $10.1101 / \mathrm{lm} .40001$

Muth, F., Cooper, T. R., Bonilla, R. F., \& Leonard, A. S. 2018. A novel protocol for studying bee cognition in the wild. Methods in Ecology and Evolution, 9(1), 78-87. DOI: 10.1111/2041-210X.12852

Nicholls, E., \& Ibarra, N. H. 2016. Assessment of pollen rewards by foraging bees. Functional Ecology, 31(1), 76-87. DOI: 10.1111/13652435.12778

Ollerton, J., Winfree, R., \& Tarrant, S. 2011. How many flowering plants are pollinated by animals? Oikos, 120(3), 321-326. DOI: 10.1111/j.16000706.2010.18644.x 
Pavlov, I. P. 1927. Conditioned reflexes. London: Oxford University Press: p. 448.

Raguso, R. A. 2008. Wake up and smell the roses: the ecology and evolution of floral scent. Annual Review of Ecology, Evolution and Systematics, 39(1), 549-569. DOI: 10.1146/annurev. ecolsys.38.091206.095601

Rath, L., Galizia, G. C., \& Szyszka, P. 2011. Multiple memory traces after associative learning in the honey bee antennal lobe. European Journal of Neuroscience, 34(2), 352-360. DOI: 10.1111/j.1460-9568.2011.07753.x

Rech, A. R., Agostini, K., Oliveira, P. E., \& Machado, I. C. 2014a. Biologia da polinização. Rio de Janeiro: Projeto Cultural: p. 527.

Rech, A. R., Bergamo, P. J., \& Figueiredo, R. A. 2014b. Polinização abiótica. In: A. R. Rech, K. Agostini, P. E. Oliveira, \& I. C. Machado (Eds.), Biologia da polinização. pp. 103-204. Rio de Janeiro: Projeto Cultural.

Rech, A. R., Dalsgaard, B., Sandel, B., Sonne, J., Svenning, J. C., Holmes, N., \& Ollerton, J. 2016. The macroecology of animal versus wind pollination: ecological factors are more important than historical climate stability. Plant Ecology and Diversity, 9(3), 253-262. DOI: 10.1080/17550874.2016.1207722

Rodríguez, I., Gumbert, A., Ibarra, N. H., Kunze, J., \& Giurfa, M. 2004. Symmetry is in the eye of the beeholder: innate preference for bilateral symmetry in flower-naïve bumblebees. Naturwissenschaften, 91(8), 374-7. DOI: 10.1007/ s00114-004-0537-5

Roselino, A. C., Rodrigues, A. V., \& Hrncir, M. 2016. Stingless bees (Melipona scutellaris) learn to associate footprint cues at food sources with a specific reward context. Journal of Comparative Physiology A: Neuroethology, Sensory, Neural, and Behavioral Physiology, 202(9-10), 657-666. DOI: $10.1007 /$ s00359-016-1104-1

Salzmann, C. C., Nardella, A. M., Cozzolino, S., \& Schiestl, F. P. 2007. Variability in floral scent in rewarding and deceptive orchids: the signature of pollinator-imposed selection? Annals of Botany, 100(4), 757-765. DOI: 10.1093/aob/mcm161

Sanchez, M. G. B. , Serre, M., Avargues-Weber, A., Dyer, A. G., \& Giurfa, M. 2015. Learning context modulates aversive taste strength in honey bees. Journal of Experimental Biology, 218(6), 949-959. DOI: 10.1242/jeb.117333
Skinner, B. F. 1938. The behavior of organisms. New York: Appleton.

Sletvold, N., Trunschke, J., Smit, M., Verbeek, J., \& Ågren, J. 2016. Strong pollinator-mediated selection for increased flower brightness and contrast in a deceptive orchid. Evolution, 70(3), 716-724. DOI: 10.1111/evo.12881

Stach, S., \& Giurfa, M. 2005. The influence of training length on generalization of visual feature assemblies in honeybees. Behavioural Brain Research, 161(1), 8-17. DOI: 10.1016/j. bbr.2005.02.008

Stavenga, D. G. 1992. Eye regionalization and spectral tuning of retinal pigments in insects. Trends in Neurosciences, 15(6), 213-8. DOI: 10.1016/0166-2236(92)90038-A

Szyszka, P., Gerkin, R. C., Galizia, C. G., \& Smith, B. H. 2014. High-speed odor transduction and pulse tracking by insect olfactory receptor neurons. Proceedings of the National Academy of Sciences, 111(47), 16925-16930. DOI: 10.1073/ pnas. 1412051111

Takeda, K. 1961. Classical conditioned response in the honey bee. Journal of Insect Physiology, 6(3), 168-179. DOI: 10.1016/0022-1910(61)90060-9

Telles, F. J., Corcobado, G., Trillo, A., \& RodríguezGironés, M. A. 2017. Multimodal cues provide redundant information for bumblebees when the stimulus is visually salient, but facilitate red target detection in a naturalistic background. PLoS ONE, 12(9), e0184760. DOI: 10.1371/ journal.pone. 0184760

Telles, F. J., \& Rodríguez-Gironés, M. A. 2015. Insect vision models under scrutiny: what bumblebees (Bombus terrestris terrestris L.) can still tell us. The Science of Nature, 102(1-2), 1256. DOI: 10.1007/s00114-014-1256-1

Turner, C. H. 1910. Experiments on color-vision of the honey bee. The Biological Bulletin, 19(5), 257-279.

Vieira, A. R., Salles, N., Borges, M., \& Mota, T. 2018. Visual discrimination transfer and modulation by biogenic amines in honeybees. Journal of Experimental Biology, 221(9), jeb178830. DOI: 10.1242/jeb. 178830

Weiss, M. R. 1991. Floral colour changes as cues for pollinators. Nature, 354(6350), 227-229. DOI: $10.1038 / 354227 \mathrm{a} 0$

Weiss, M. R. 2001. Vision and learning in some neglected pollinators: beetles, flies, moths, 
and butterflies. In: L. Chittka \& J. D. Thomson (Eds.), Cognitive ecology of pollination: animal behavior and floral evolution. pp. 171-190. New York: Cambridge University Press.

Weiss, M.R., \&Lamont, B. B. 1997. Floral color change and insect pollination: a dynamic relationship. Israel Journal of Plant Sciences, 45(2-3), 185199. DOI: 10.1080/07929978.1997.10676683

Wester, P., \& Lunau, K. 2017. Plant-pollinator communication. Advances in Botanical Research, 82(1), 225-257. DOI: 10.1016/ bs.abr.2016.10.004

Willmer, P. 2011. Pollination and floral ecology. Princeton: Princeton University Press: p. 792.

Material Suplementar 1. Glossário de termos técnicos.

Supplementary Material 1. Glossary of technical terms.

Submitted: 05 February 2019 Accepted: 03 September 2019 Published online: 03 September 2019 Associate Editor: Leandro dos Santos Lima Hohl 